Aldo L. Schenone, MD

Non-Invasive Cardiovascular Imaging

Department, Brigham and Women's

Hospital, Boston, MA
A. Michael Lincoff, MD

Department of Cardiovascular Medicine; Heart, Vascular

and Thoracic Institute; Director, C5Research (Cleveland Clinic

Coordinating Center for Clinical Research); Director, Center for

Clinical Research, Lerner Research Institute, Cleveland Clinic;

Professor of Medicine, Cleveland Clinic Lerner College

of Medicine of Case Western Reserve University, Cleveland, $\mathrm{OH}$

\title{
Aspirin for primary prevention of atherosclerotic cardiovascular events
}

\section{ABSTRACT}

Recent trials evaluated the impact of aspirin for primary prevention of cardiovascular events in patients at intermediate risk, patients with diabetes, and the elderly, and the results have been incorporated into the most recent professional guidelines. For the most part, the role of aspirin in primary prevention remains limited, albeit not adequately tested in those at higher risk.

\section{KEY POINTS}

Using aspirin for the sole purpose of primary prevention is discouraged in healthy elderly patients (age $>70$ ) and those at high risk of bleeding, including patients on anticoagulation.

Routine use of aspirin for the sole purpose of primary prevention is best deferred in patients at low risk $(<5 \%$ 10 -year risk) and borderline risk (5\%-7.5\% 10-year risk). However, in selected patients at borderline risk, such as those with a strong family history, clinicians could also consider risk-enhancers such as coronary calcium.

A shared decision to initiate aspirin in those at intermediate risk (7.5\%-20\% 10-year risk) could be considered for patients with a risk-enhancer such as an elevated coronary calcium score (> 100) or elevated lipoprotein(a) (> $50 \mathrm{mg} / \mathrm{dL}$ ).

In patients age 40 to 70 at high risk (> 20\% at 10 years), it is reasonable to consider starting low-dose aspirin as a shared decision if the patient is thought to be at low risk of bleeding.

doi:10.3949/ccjm.87a.19045
SPIRIN Is ONE OF the most widely used drugs A in cardiovascular medicine, with one-third of adults over the age of 40 using it for either primary or secondary prevention of major cardiovascular events. But does this widespread use achieve the intended goal of reducing the incidence of cardiovascular events and death?

The use of aspirin undoubtedly reduces the risk of a subsequent cardiovascular event or death from vascular causes when used for secondary prevention - eg, in patients who have had a myocardial infarction, stroke, or symptomatic peripheral arterial disease or who have undergone coronary revascularization. ${ }^{1-5}$ However, its net impact in primary prevention (ie, in patients without established cardiovascular disease or previous coronary revascularization) has been debated for years. ${ }^{5-7}$

Recent clinical trials have reevaluated the role of aspirin in primary prevention. ${ }^{8-12}$ The results suggest that aspirin should play a more limited role than in the past, and this evidence has resulted in an update in the recommendations from the American Heart Association (AHA) and American College of Cardiology (ACC). ${ }^{13}$

This review examines the evidence on the risk-benefit profile of aspirin in primary prevention of cardiovascular events (Table 1), 8-12,14-24 summarizes current recommendations on this topic, and proposes an evidence-based algorithm to guide the use of aspirin for primary prevention in clinical practice.

\section{CONFLICTING EVIDENCE FROM OLDER TRIALS}

\section{ATC meta-analysis, 2009}

The Anti-thrombotic Trialists' Collaboration (ATC) meta-analysis, published in 2009, was a landmark study of the role of aspirin in pri- 


\section{TABLE 1}

\section{Trials of aspirin as primary prevention}

\begin{tabular}{|c|c|c|c|c|c|}
\hline \multirow[b]{2}{*}{ Trial } & \multirow[b]{2}{*}{ Year } & \multirow[b]{2}{*}{ Population } & \multicolumn{3}{|c|}{ Number needed to treat or harma } \\
\hline & & & $\begin{array}{l}\text { Nonfatal } \\
\text { myocardial } \\
\text { infarction }\end{array}$ & $\begin{array}{l}\text { Nonfatal } \\
\text { ischemic } \\
\text { stroke }\end{array}$ & $\begin{array}{l}\text { Major } \\
\text { gastro- } \\
\text { intestinal } \\
\text { bleed }\end{array}$ \\
\hline BDS $^{14}$ & 1988 & 5,139 healthy male physicians & [143] & [250] & ND \\
\hline PHS $^{15}$ & 1989 & 22,071 healthy male physicians & 67 & [333] & [250] \\
\hline TPT $^{16}$ & 1998 & 5,085 men at high risk & 40 & 125 & [250] \\
\hline HOT $^{17}$ & 1998 & 18,790 people with hypertension & 77 & 1,000 & [71] \\
\hline $\mathbf{P P P}^{19}$ & 2003 & 4,495 people with risk factors & 143 & 250 & 333 \\
\hline WHS $^{18}$ & 2005 & 39,876 healthy female nurses & ND & 500 & 1,000 \\
\hline POPADAD $^{21}$ & 2008 & 1,276 people with diabetes, $A B I \leq 0.99$ & 500 & 36 & 143 \\
\hline JPAD ${ }^{23}$ & 2008 & 2,539 people with diabetes & [167] & 1,000 & [200] \\
\hline $\mathbf{A A A}^{24}$ & 2010 & 3,350 people with $A B I \leq 0.91$ & 200 & 1,000 & {$[1,000]$} \\
\hline JPPP 22 & 2014 & 14,000 people with $\geq 1$ risk factor & 250 & ND & [50] \\
\hline ARRIVE $^{8}$ & 2018 & $\begin{array}{l}12,526 \text { men with } 2-4 \text { risk factors or } \\
\text { women with } \geq 3 \text { risk factors }\end{array}$ & 333 & 333 & [100] \\
\hline ASCEND $^{9}$ & 2018 & 15,480 people with diabetes & 1,000 & 333 & [167] \\
\hline ASPREE $^{10-12}$ & 2018 & 19,114 healthy elderly & 333 & 250 & 42 \\
\hline
\end{tabular}

aThe number of patients who would need to be treated for 10 years to prevent or cause 1 event, calculated as the inverse of the absolute difference in the proportion of patients with events per year between the aspirin and placebo groups. Numbers in brackets indicate harm, ie, higher rates in the aspirin group.

$\mathrm{AAA}=$ Aspirin for Asymptomatic Atherosclerosis; $\mathrm{ABI}=$ ankle-brachial index; $\mathrm{ASCEND}=\mathrm{A}$ Study of Cardiovascular Events in Diabetes; ASPREE = A Study of Cardiovascular Events in Diabetes (ASCEND) and Aspirin in Reducing Events in the Elderly; ARRIVE = Aspirin to Reduce Risk of Initial Vascular Events; BDS = British Doctors Study; HOT = Hypertension Optimal Treatment; JPAD = Japanese Primary Prevention of Atherosclerosis With Aspirin for Diabetes; JPPP = Japanese Primary Prevention Project; ND = no difference; PHS = Physicians' Health Study; POPADAD = Prevention of Progression of Arterial Disease and Diabetes; PPP $=$ Primary Prevention Project; TPT $=$ Thrombosis Prevention Trial; WHS = Women's Health Study

Aspirin is one of the most widely used drugs in cardiovascular medicine mary prevention. ${ }^{5}$ It analyzed patient-level data from 6 trials published between 1988 and $2005,{ }^{14-19}$ mostly addressing the impact of aspirin in patients at low risk, with an event rate of about $0.5 \%$ per year (or $5 \%$ in 10 years).

Serious vascular events (myocardial infarction, stroke, and vascular death) occurred at a rate of $0.51 \%$ per year in the aspirin groups and $0.57 \%$ in the placebo groups. Although this represented a $12 \%$ reduction in relative risk (relative risk $[\mathrm{RR}]$ 0.88, 95\% confidence interval [CI] 0.82-0.94, $P=.0001$ ), the absolute risk reduction was very modest: 1,667 patients would need to be treated for 1 year (or 167 for 10 years) to prevent 1 major cardiovascular event. This re- duction was primarily driven by a reduction in nonfatal myocardial infarctions and ischemic strokes but not in vascular mortality.

Although all the prespecified subgroups showed a similar proportional reduction in major cardiovascular events, the data suggested that men and women might benefit differently from aspirin. Men primarily experienced a reduction in nonfatal myocardial infarctions, while women benefited from a reduction in ischemic strokes. ${ }^{5,20}$ However, this differential effect was not noted in the secondary prevention cohort of the meta-analysis and was no longer statistically significant when accounting for multiple comparisons. 
The modest reduction in nonfatal cardiovascular events came at the cost of excess bleeding - and 1 in every 4 events was a hemorrhagic stroke. Major extracranial bleeding occurred in $0.10 \%$ of the aspirin recipients and $0.07 \%$ of the placebo recipients, and hemorrhagic stroke occurred in $0.04 \%$ vs $0.03 \%$, for an increase of $0.04 \%$ per year in the rate of major bleeding. Thus, 1 major bleeding event would be caused after treating 333 patients for 10 years. Based on this evidence, 2 nonfatal cardiovascular events would be prevented per major bleed caused.

Hence, the net impact of aspirin for primary prevention of major cardiovascular events was at best marginal in these earlier trials, mainly reducing the incidence of nonfatal cardiovascular events and largely counterbalanced by the risk of bleeding.

Also, at the time these data were gathered, best preventive practices and aspirin dosing significantly differed from the current standard of care. Today, tighter blood pressure control, lower rates of smoking, and widespread use of statins likely make the risk-benefit profile of aspirin in primary prevention less favorable. For instance, using a statin halves the benefit of aspirin without attenuating the bleed-

Aspirin would cause about

1 major bleeding event

for every 2 cardiovascular events prevented ing risk, which could completely dissipate the marginal benefit reported in the ATC metaanalysis and perhaps render aspirin harmful in patients at low risk. ${ }^{5}$

Despite the very modest benefits reported in these early trials, debate continued as to whether the net impact would be more favorable in patients at higher risk of cardiovascular events. Evidence supporting this notion was that the relative risk reduction in major cardiovascular events was about the same in both primary prevention (RR $0.88,95 \% \mathrm{CI}$ $0.82-0.94$ ) and secondary prevention (RR $0.81,95 \%$ CI $0.82-1.00) .{ }^{5}$ For context, giving aspirin in the secondary prevention cohort resulted in a $1.5 \%$-per-year absolute reduction (number needed to treat 7 for 10 years) in the annual rate of major cardiovascular events compared with the marginal absolute reduction of $0.07 \%$ per year (number needed to treat 167 for 10 years) in the primary prevention cohort. This difference in absolute benefit is due to the substantially higher baseline rate of cardiovascular events in the secondary prevention group.

\section{Further studies, 2008-2014}

Hence, a second wave of studies, published between 2008 and 2014, sought to evaluate the impact of aspirin for primary prevention in a higher-risk population, ie, patients with diabetes, low ankle-brachial index, or other cardiovascular risk factors. ${ }^{21-24}$ Despite this intention, these trials, like the earlier ones, largely studied a low-risk population. Only in the Prevention of Progression of Arterial Disease and Diabetes trial was the event risk greater than $1 \%$ per year (or $>10 \%$ in 10 years). ${ }^{21}$

Most of these studies were small and therefore lacked the power to detect differences in cardiovascular event rates, with only the Japanese Primary Prevention Project having a sample size comparable to the earlier trials. ${ }^{22}$

Collectively, these studies failed to show any benefit of aspirin for primary prevention of major cardiovascular events, and even the betterpowered Japanese Primary Prevention Project was stopped early due to futility regarding the composite end point of death, myocardial infarction, and stroke. Although an overall negative trial, this was the only study from 2008 to 2014 to report a potential reduction in nonfatal myocardial infarction as a secondary end point, a suggested benefit that was largely counterbalanced by a higher rate of major bleeding. ${ }^{22}$

The negative results of these studies came as a surprise and greatly contrasted with the at-least marginal benefit reported by the earlier trials. These results were attributed in part to lack of statistical power but also to improved management of cardiovascular risk factors with tighter blood pressure control, smoking cessation, and statin use.

To overcome the lack of power of these trials, an updated meta-analysis was conducted, pooling all available studies from 1988 through 2014, which replicated the finding of marginal benefit of aspirin reported in the ATC analysis. Yet the results were mainly driven by studies published before 2005, with no benefit found in the later trials. ${ }^{25,26}$

\section{CONTEMPORARY TRIALS: THE TIEBREAKER?}

Three recent clinical trials examined the role of aspirin for primary prevention of major cardiovascular events in a contemporary cohort 
of patients thought to be at higher risk of cardiovascular events in 3 distinct populations:

- Patients at moderately elevated risk of cardiovascular events (estimated 10-year risk $10 \%-20 \%)^{8}$

- Patients with diabetes ${ }^{9}$

- The elderly. ${ }^{10-12}$

\section{The ARRIVE trial:} No benefit in patients at low risk

The use of aspirin for primary prevention of cardiovascular events in those with elevated risk has been a point of heated debate in clinical practice.

The ASA to Reduce Risk of Initial Vascular Event (ARRIVE) trial $^{8}$ was designed to clarify the benefit of aspirin in patients with an estimated 10 -year risk of $10 \%$ to $20 \%$. However, the actual rate of events was lower, at about $0.8 \%$ per year (or $8 \%$ in 10 years). Due to the low rate of events, the original primary end point (myocardial infarction, stroke, and cardiovascular death) had to be modified to include unstable angina and transient ischemic attack to adequately power the study.

The incidence of the primary composite end point and those of each component of the composite end point was no different between the aspirin and control groups. However, aspirin was associated with a significant reduction in myocardial infarction on per-protocol analysis. This finding must be interpreted with caution, given the potential for bias associated with a per-protocol analysis, although it provides an opportunity to explore the impact of aspirin in those that completed the intended intervention.

On the other hand, the use of aspirin was associated with a 2 -fold higher rate of gastrointestinal bleeding $(0.97 \%$ vs $0.46 \%, P=$ .0007 ), predominantly mild gastrointestinal bleeding. Approximately 1 gastrointestinal bleeding event would be caused by treating 196 patients for 10 years. Importantly, the incidence of bleeding was likely underestimated in this study, given the exclusion of patients perceived to be at higher risk of bleeding at enrollment.

In summary, this study found aspirin to have an unfavorable risk-benefit profile when used for primary prevention of cardiovascular events in a contemporary low-risk cohort. In this patient population, aspirin offers no adjunctive cardiovascular preventive benefit and moreover increases the risk of bleeding. Whether the cardiovascular benefit of aspirin in a higher-risk (> 10\%-20\% estimated 10 year risk) population without diabetes outweighs the bleeding risk remains unknown.

\section{The ASCEND trial:}

Modest benefit in patients with diabetes

Preventive use of aspirin in patients with diabetes without established atherosclerotic cardiovascular disease has been another point of controversy. Patients with diabetes suffer a 2 to 3-fold higher rate of cardiovascular events, and they are thus thought to be a population that could benefit from preventive use of aspirin. Although earlier trials in patients with diabetes failed to demonstrate a definite benefit of aspirin in this group, these studies were grossly underpowered.

The larger Study of Cardiovascular Events in Diabetes (ASCEND) trial $^{9}$ assessed the benefit of aspirin in men and women age 40 or older with diabetes and without atherosclerotic cardiovascular disease. Once again, the event rate was lower than anticipated, with an annual risk of $1.25 \%$ to $1.3 \%$ (or estimated 10 year risk of $12 \%-13 \%$ ). As a result, the sample size and follow-up had to be increased, and the primary composite end point was modified to include transient ischemic attack to maintain the intended power.

Preventive use of aspirin resulted in an absolute reduction of $0.17 \%$ per year in the rate of the composite end point of nonfatal myocardial infarction, nonfatal stroke, transient ischemic attack, and vascular death (hazard ratio [HR] 0.88, 95\% CI 0.79-0.97, $P=.01$ ). A total of 59 patients would need to be treated for 10 years to prevent 1 major cardiovascular event.

However, there was an absolute annual excess in major bleeding of $0.13 \%$ per year (HR $1.29,95 \%$ CI $1.09-1.52, P=.003)$, which included gastrointestinal $(62.3 \%)$, ocular $(21.1 \%)$, intracranial $(17.2 \%)$, and other site (20.4\%) bleeds. Thus, 1 major bleeding event would be caused after treating 77 patients for 10 years. Thus, the use of low-dose aspirin in patients with diabetes ( $>10 \%$ risk at 10 years) led to a modest reduction in cardiovascular

\section{Statins may dilute the potential benefit of aspirin}


events that was largely offset by an increase in major bleeding.

\section{The ASPREE trial:} Aspirin was harmful in the elderly

Similarly, the elderly are a population at increased risk of cardiovascular events that could, it was thought, profit from preventive use of aspirin.

The ASA in Reducing Events in the Elderly (ASPREE) trial sought to define the role of aspirin in those 70 years or older (or 65 or older if Hispanic or black) without established atherosclerotic cardiovascular disease and having a life expectancy longer than 5 years. ${ }^{10-12}$ Those with a clinical diagnosis of dementia, substantial physical disability, or high bleeding risk were excluded, as were those adhering to their medications less than $80 \%$ of the time during a run-in period. The main goal of the study was to determine if aspirin $100 \mathrm{mg}$ daily would prolong a healthy life span in this population, using the composite end point of death, dementia, and persistent physical disability.

The study was stopped early due to futility after a mean follow-up of 4.7 years. There

Many questions remain about the use of aspirin for primary prevention of atherosclerotic cardiovascular disease were no differences in the rates of the primary end point or individual secondary end points of cardiovascular events, dementia, or disability between the treatment groups. ${ }^{10,12}$

Worse, the use of aspirin translated into a net excess of $0.24 \%$ per year in major bleeding events (HR 1.38, 95\% CI 1.18-1.62, P $<.001$ ), resulting in 1 major bleeding event after treating 42 patients for 10 years. Again, this risk is likely underestimated, given the exclusion of patients who could not tolerate aspirin during the run-in period. Aspirin increased the rates of upper gastrointestinal bleeds (HR 1.87, 95\% CI 1.32-2.66) and intracranial hemorrhage of any kind (HR 1.50, 95\% CI 1.11-2.02).

Surprisingly, a higher rate of all-cause mortality (HR 1.14, 95\% CI 1.01-1.29 was noted in the aspirin group, mainly driven by cancer death, particularly colorectal cancers, challenging the theory that aspirin could prevent this type of cancer.

In summary, this contemporary primary prevention trial demonstrated a rather deleterious effect of aspirin in an unselected healthy elderly population (age $>70$ ).

A new meta-analysis: Modest benefit, unacceptable risk

A 2019 meta-analysis ${ }^{27}$ summarized all available evidence, including the contemporary trials.

The use of aspirin for primary prevention of atherosclerotic cardiovascular disease was associated with a $0.3 \%$-per-year reduction in the absolute risk of myocardial infarction but no benefit in reducing the risk of stroke or death, a very modest benefit that disappears when analyzing only studies published after 2008. Further, aspirin use in primary prevention is consistently associated with an absolute increase in the rates of intracranial bleeding at $0.1 \%$ per year and major bleeding at $0.2 \%$ per year. ${ }^{27}$

Overall, the use of aspirin appears harmful when prescribed for primary prevention of cardiovascular events in lower-risk patients without diabetes and unselected healthy elderly populations (age $>70$ ). In patients without diabetes, a total of 265 patients need to be treated with aspirin for 10 years to prevent 1 cardiovascular event, while 1 major bleeding event would be caused after treating 210 patients.

On the other hand, patients with diabetes without atherosclerotic cardiovascular disease appear to enjoy a modest reduction in cardiovascular events when prescribed aspirin for primary prevention, although this benefit is largely counterbalanced by an increase in bleeding. About 153 diabetic patients would need to be treated for 10 years to prevent 1 major cardiovascular event, while treating 121 patients for 10 years would cause 1 major bleeding event.

Many questions remain regarding the use of aspirin for primary prevention of atherosclerotic cardiovascular disease. For instance it is still largely unknown whether it is justified in a higher-risk primary prevention cohort (> $20 \%$ estimated 10 -year risk), or those with uncontrolled risk factors or risk enhancers such as coronary calcium, elevated lipoprotein(a), or elevated inflammatory markers.

\section{PAST GUIDELINE UNCERTAINTIES AND CURRENT RECOMMENDATIONS}

Major cardiovascular societies have interpreted the available evidence differently, leading to discrepant recommendations on the use of 


\section{SCHENONE AND LINCOFF}

\section{TABLE 2}

\section{Aspirin for primary prevention: Recommendations from major societies}

\section{American Heart Association/American College of Cardiology, 2002 ${ }^{7}$}

Low-dose aspirin recommended in persons at higher cardiovascular risk, especially those with 1-year risk $\geq 10 \%$

Low-dose aspirin recommended in patients with diabetes at increased cardiovascular risk, including those who are over age 40 or who have additional risk factors

Therapy should not be recommended for patients with diabetes under age 21 because of the increased risk of Reye syndrome associated with aspirin use in this population; patients with diabetes under age 30 have not been studied

\section{European Society of Cardiology, $2016^{6}$}

Antiplatelet therapy is not recommended in individuals without cardiovascular disease due to the increased risk of major bleeding Antiplatelet therapy (eg, aspirin) is not recommended for people with diabetes who do not have cardiovascular disease

\section{US Preventive Services Task Force, 2017}

Low-dose aspirin is recommended in adults ages $50-59$ who have $a \geq 10 \% 10$-year risk, are not at increased risk for bleeding, have a life expectancy of $\geq 10$ years, and are willing to take it daily for $\geq 10$ years

The decision to initiate low-dose aspirin for primary prevention of cardiovascular disease and colorectal cancer in adults ages 60-69 who have a $10 \%$ or greater 10 -year cardiovascular disease risk should be an individual one

The current evidence is insufficient to assess the balance of benefits and harms of initiating aspirin use for the primary prevention of cardiovascular disease and colorectal cancer in adults under age 50

The current evidence is insufficient to assess the balance of benefits and harms of initiating aspirin use for primary prevention of cardiovascular disease and colorectal cancer in adults age 70 or older

\section{American Academy of Family Physicians, 2016 33}

Low-dose aspirin use for primary prevention of cardiovascular disease and colorectal cancer is recommended in adults ages 50-59 who have a 10\% or greater 10-year cardiovascular disease risk, are not at increased risk for bleeding, have a life expectancy of at least 10 years, and are willing to take low-dose aspirin daily for at least 10 years

The decision to initiate low-dose aspirin use for the primary prevention of cardiovascular disease and colorectal cancer in adults ages 60-69 who have a $10 \%$ or greater 10-year cardiovascular disease risk should be an individual one

Evidence is insufficient to assess risk-benefit profile of aspirin for primary prevention of cardiovascular disease and colorectal cancer in adults younger than 50

The current evidence is insufficient to assess the balance of benefits and harms of starting aspirin for primary prevention of cardiovascular disease and colorectal cancer in adults age 70 or older

\section{American Diabetes Association, 2018 ${ }^{32}$}

Low-dose aspirin may be considered as a primary prevention strategy in those with type 1 or type 2 diabetes who are at increased cardiovascular risk; this includes most men and women with diabetes age $>50$ who have at least 1 additional major risk factor and are not at increased risk of bleeding

\section{American Heart Association/American College of Cardiology, 201913}

Low-dose aspirin might be considered for the primary prevention of atherosclerotic cardiovascular disease (ASCVD) among select adults ages 40-70 who are at higher ASCVD risk but not at increased bleeding risk

Low-dose aspirin should not be prescribed on a routine basis for primary prevention of ASCVD among adults age $>70$ Low-dose aspirin should not be prescribed for primary prevention of ASCVD among adults of any age who are at increased risk of bleeding 
aspirin in primary prevention (Table 2). 6,13,28-34

Earlier guidelines from the AHA/ACC, US Preventive Services Task Force (USPSTF), and American Diabetes Association supported the use of aspirin for primary prevention of cardiovascular events in those at high risk. ${ }^{28-32}$ The USPSTF and AHA/ACC used an estimated 10 -year risk higher than $10 \%$ as a criterion for prophylactic initiation of aspirin. This recommendation was based on post hoc analysis of older trials that suggested with moderate certainty that the risk-benefit profile of aspirin was more favorable in those with an estimated 10 -year risk greater than $10 \%$, particularly in those age 50 to 59 .

In contrast, the 2016 European Society of Cardiology guidelines ${ }^{6}$ advised against using aspirin for primary prevention, even before the 3 newer trials described above were published. This recommendation was based on a more direct interpretation of the evidence, acknowledging that even if aspirin conferred a very modest reduction in nonfatal cardiovascular events, this was largely offset by an increase in bleeding, with no decrease in mortality.

AHA/ACC 2019. Most recently, the

The decision to start aspirin should be individualized and shared AHA/ACC updated their recommendations, ${ }^{13}$ considering the evidence from the newer trials. The 2019 AHA/ACC guidelines recognized the greatly attenuated benefit of adjunctive aspirin in contemporary optimal medical management and acknowledged the systematic overestimation of risk with the use of pooled cohort equations. Hence, they downgraded the class of recommendation for prophylactic use of aspirin to class IIb ("it may be considered") and removed any specific pooled cohort equation risk levels as criteria for recommending aspirin. In the new guidelines, prophylactic use of aspirin may be considered in selected adults age 40 to 70 at higher risk of atherosclerotic cardiovascular disease without a higher bleeding risk. In contrast, routine use of aspirin in healthy elders age 70 or older and in those at high bleeding risk is discouraged.

These more cautious recommendations highlight the lingering uncertainty about the impact of this intervention in those at the higher end of the cardiovascular risk spectrum such as those with uncontrolled risk factors despite optimal medical management, those with subclinical coronary atherosclerosis, and those with additional risk-enhancing factors such as elevated inflammatory markers or elevated lipoprotein(a).

Ultimately, the guidelines defer the decision to initiate aspirin for primary prevention to the patient-clinician encounter after weighing the risk vs the possible benefit in the patient in question and the totality of the evidence available. ${ }^{13}$

\section{REMAINING GAPS IN KNOWLEDGE}

As previously mentioned, most of the available evidence pertains to a low-risk primary prevention cohort, and no high-quality study has been conducted to assess the net risk-benefit profile of aspirin in selected young patients (age $<40$ ) or in high-risk patients (> 20\% 10year risk) such as those with uncontrolled risk factors or risk enhancers. Therefore, great uncertainty remains about the potential impact of aspirin in selected young patients and those thought to be at the higher end of the risk spectrum.

Patients at high risk. Although a larger absolute benefit would be expected in those at higher baseline cardiovascular risk, it is also anticipated that the bleeding risk will increase, given the concomitant increase in bleeding associated with several cardiovascular risk factors such old age, diabetes, obesity, and smoking. ${ }^{30}$ Whether these risk factors increase cardiovascular disease and bleeding risk by the same magnitude remains unknown. Hence, the patient profile and the specific risk cutoff at which the primary prevention benefits of aspirin outweigh the bleeding risk remain unknown.

Another area of ambiguity relates to the proper classification (primary vs secondary prevention cohort) and subsequent management of patients without symptoms who are found incidentally to have atherosclerotic cardiovascular disease on coronary angiography or noninvasive imaging such as coronary artery calcium scoring or coronary computed tomographic angiography.

Coronary calcium. It is well known that the total burden of coronary plaque directly correlates with the rate of cardiovascular 
events. Such plaque burden can be easily estimated by measuring coronary artery calcium, with higher calcium scores resulting in proportional increment in cardiovascular risk.

Therefore, measuring coronary calcium greatly enhances the accuracy in estimating risk of cardiovascular events irrespective of age and comorbidities. ${ }^{35}$ For instance, patients with a calcium score greater than 100 experience cardiovascular events at a rate close to that in a stable secondary prevention population, while those with extensive calcium (scores $>1,000$ ) experience event rates that exceed the rates observed in secondary prevention trials. ${ }^{36,37}$ On the other hand, absence of coronary calcium (scores of 0 ) is equally helpful in establishing that patients are not at risk, which is particularly helpful in those with a borderline or intermediate risk estimation based on the pooled cohort equation..$^{38}$

Evidence from the Multi-ethnic Study of Atherosclerosis (MESA) ${ }^{39}$ suggested that the coronary calcium score might be of value when deciding whether to start aspirin for primary prevention of cardiovascular disease in patients without diabetes. In this regard, the risk-benefit profile of aspirin appears favorable in those with a calcium score greater than 100 , with the odds of preventing vascular events 2 to 4 times higher than the chance of causing a bleed. In contrast, aspirin seems harmful in those with a score of 0 , with a chance of bleeding that is 2 to 4 times higher than the likelihood of preventing a vascular event, regardless of traditional risk factors.

Lipoprotein(a). Similarly, the utility of lipoprotein(a) to ascertain the benefit of aspirin for the primary prevention of cardiovascular events remains uncertain. In a substudy from the Women's Health Study, ${ }^{40}$ an elevated lipoprotein(a) was associated with a 2-fold higher rate of cardiovascular events, which was effectively attenuated by the use of aspirin.

High-quality studies are needed to define the role of coronary calcium and lipoprotein(a) in the decision to start aspirin for primary prevention of atherosclerotic cardiovascular disease in patients both with and without diabetes.
Aspirin dose. The optimal aspirin dose for primary prevention events remains uncertain. The current understanding is that low doses (75-100 mg per day) are effective in preventing vascular events while minimizing bleeding rates. On the other hand, the impact of statins and proton-pump inhibitors on the risk-benefit profile of aspirin in primary prevention remains unresolved. ${ }^{41}$ Further studies are needed to evaluate the optimal aspirin dosing. The upcoming ASA and Simvastatin Combination for Cardiovascular Events Prevention Trial (ACCEPT-D) is set to evaluate the strongly suspected attenuation effect of statins on the aspirin benefit. ${ }^{42}$

\section{FROM THE EVIDENCE TO THE PATIENT}

The decision to defer or prescribe aspirin in clinical practice for primary prevention of cardiovascular events remains a challenging one and should be individualized. It is important to first emphasize that primary prevention recommendations only apply to those patients without established atherosclerotic cardiovascular disease, namely no prior myocardial infarction, no prior ischemic stroke, no symptomatic peripheral arterial disease, and no prior coronary revascularization. In patients with these conditions, ie, the secondary prevention cohort, the benefit of aspirin is well established.

Primary prevention should always begin with encouragement of healthy life habits and optimal management of cardiovascular risk factors including weight loss, glucose and blood pressure control, and lipid management, per preventive guideline recommendations. ${ }^{13}$

The risk of atherosclerotic cardiovascular disease should be estimated using the pooled cohort equation in every patient before deciding on the prophylactic use of aspirin. In the new guidelines, patients are classified into 4 risk categories based on the pooled cohort equation ${ }^{13}$ :

- Low risk $(10$-year risk $<5 \%)$

- Borderline risk ( $5 \%$ to $<7.5 \%$ )

- Intermediate risk (7.5\% to $<20 \%)$

- High risk ( $\geq 20 \%)$.

Of importance, the current risk estimation tools (including the 10-year pooled cohort equation) systematically overestimate risk.
Primary prevention should always begin with encouragement of healthy life habits and optimal management of cardiovascular risk factors 


\section{Aspirin for primary prevention of cardiovascular disease}

No history of myocardial infarction, ischemic stroke, or symptomatic peripheral arterial disease

No previous coronary revascularization

$\downarrow$

Emphasize adherence to a healthy lifestyle (eg, Mediterranean diet, physical activity)

Achieve optimal risk factor modification (eg, blood pressure control, glucose control, lipid management, weight loss)

\begin{tabular}{|c|c|c|c|}
\hline Age $<40$ & $\begin{array}{l}\text { Patients without diabetes } \\
\text { Age } 40-70\end{array}$ & Age $>70$ & Patients with diabetes \\
\hline $\begin{array}{l}\text { Insufficient evidence to guide } \\
\text { the use of aspirin }\end{array}$ & $\begin{array}{l}\text { Estimate pooled risk }{ }^{\mathrm{a}} \text { and risk } \\
\text { of bleeding }\end{array}$ & \multirow{2}{*}{$\begin{array}{l}\text { Do not start aspirin for the } \\
\text { sole purpose of reducing vas- } \\
\text { cular events in the otherwise } \\
\text { healthy elderly }\end{array}$} & $\begin{array}{l}\text { on the risk and benefit of } \\
\text { aspirin initiation }\end{array}$ \\
\hline $\begin{array}{l}\text { Strongly recommend risk-ben- } \\
\text { efit discussion in those at high } \\
\text { risk of ASCVD, considering b: } \\
\text { - Elevated lifetime risk of } \\
\text { ASCVD } \\
\text { - Strong family history of } \\
\text { ASCVD } \\
\text { - Personal history of familial } \\
\text { hypercholesterolemia } \\
\text { - Elevated CAC (> }>00) \\
\text { - Elevated Lp(a) (>50 mg/dL) }\end{array}$ & $\begin{array}{l}\text { Defer aspirin use if high risk of } \\
\text { bleeding }\end{array}$ & & $\begin{array}{l}\text { Consider low-dose aspirin } \\
\text { in the presence of multiple } \\
\text { risk factors, CAC }>100 \text {, or } \\
L p(a)>50 \mathrm{mg} / \mathrm{dL}\end{array}$ \\
\hline $\begin{array}{l}\text { Low risk } \\
\text { (<5\% 10-year risk of ASCVD) }\end{array}$ & $\begin{array}{l}\text { Borderline risk } \\
\text { (5\% to }<7.5 \% \text { 10-year risk) }\end{array}$ & $\begin{array}{l}\text { Intermediate risk } \\
\text { ( } 7.5 \% \text { to }<20 \% 10 \text {-year risk) }\end{array}$ & $\begin{array}{l}\text { High risk } \\
\text { ( } 220 \% \text { 10-year risk) }\end{array}$ \\
\hline \multirow[t]{6}{*}{ Defer starting aspirin } & Patient-clinician discussion & Patient-clinician discussion & Patient-clinician discussion \\
\hline & \multirow{3}{*}{$\begin{array}{l}\text { Defer aspirin initiation } \\
\text { In selected cases (eg, strong } \\
\text { family history of ASCVD), con- } \\
\text { sider further risk stratification } \\
\text { with CAC and Lp(a) to guide } \\
\text { aspirin initiation: }\end{array}$} & $\begin{array}{l}\text { Consider further risk stratifica- } \\
\text { tion with CAC and Lp(a) to } \\
\text { guide aspirin initiation: }\end{array}$ & \multirow[t]{5}{*}{$\begin{array}{l}\text { Consider low-dose aspirin, } \\
\text { especially with elevated } \\
\text { Lp(a) or CAC }\end{array}$} \\
\hline & & $\begin{array}{l}\text { CAC }>100 \text { - low-dose aspirin } \\
\text { CAC } 0 \text {-no aspirin }\end{array}$ & \\
\hline & & \multirow{3}{*}{$\begin{array}{l}\mathrm{Lp}(\mathrm{a})>50 \mathrm{mg} / \mathrm{dL} \text {-low-dose } \\
\text { aspirin } \\
\mathrm{Lp}(\mathrm{a})<50 \mathrm{mg} / \mathrm{dL} \text {-no aspirin }\end{array}$} & \\
\hline & $\begin{array}{l}\text { CAC }>100 \text { - low-dose aspirin } \\
\text { CAC } 0 \text { - no aspirin }\end{array}$ & & \\
\hline & $\begin{array}{l}\mathrm{Lp}(\mathrm{a})>50 \mathrm{mg} / \mathrm{dL} \text {-low-dose } \\
\text { aspirin } \\
\mathrm{Lp}(\mathrm{a})<50 \mathrm{mg} / \mathrm{dL} \text {-no aspirin }\end{array}$ & & \\
\hline
\end{tabular}

${ }^{a}$ Consider the consistent overestimation of cardiovascular risk by current scoring systems.

${ }^{\mathrm{b}}$ Defer aspirin in patients with increased risk of bleeding.

ASCVD = atherosclerotic cardiovascular disease; $C A C=$ coronary artery calcium score; $L p(a)=$ lipoprotein(a)

Figure 1. Our recommendations for aspirin use for primary prevention of cardiovascular events. 
Hence, incorporation of risk enhancers (eg, positive family history, elevated inflammatory markers, elevated lipoprotein(a), coronary calcium) could further refine the accuracy of the risk estimation and guide the escalation of preventive measures in selected cases. Although the use of such risk enhancers was mainly designed to guide statin therapy, they may also guide the decision to initiate aspirin in primary prevention, particularly coronary calcium and lipoprotein(a) based on available data as described above (Figure 1).

Ultimately, a preventive regimen of aspirin must reduce the rate of cardiovascular events by an absolute magnitude that is clinically meaningful, emphasizing the focus on treating those patients at the higher end of the cardiovascular risk spectrum.

It is equally important to estimate the risk of bleeding when considering primary preventive use of aspirin. Such risk might vary among patients based on age, concomitant medications, and comorbidities. ${ }^{5}$ The use of aspirin in primary prevention confers a $1.0 \%$ risk of bleeding over a 5-year period in men and a $1.1 \%$ risk in women. Age, smoking history, and diabetes, as well as a history of previous bleeding, peptic ulcer disease, cancer, and use of nonsteroidal anti-inflammatory drugs are associated with a further increase in the risk of bleeding. ${ }^{43}$ Similarly, it is well established that concomitant use of aspirin and anticoagulation is associated with a significant increase in bleeding with no ischemic benefit in a wide range of scenarios including primary prevention and atrial fibrillation. ${ }^{44,45}$

In summary, giving aspirin for primary prevention is to be considered only when the estimated cardioprotective effects of aspirin outweigh the bleeding risk on therapy.

The following recommendations are meant to provide a general guidance on the use of aspirin in clinical practice. However, the ultimate decision on whether to start or defer aspirin for primary prevention must be shared, considering the individual risk-benefit profile and the preferences of the patient at hand.

- The use of aspirin for the sole purpose of primary prevention is discouraged in healthy elderly patients (age > 70) and those at high risk of bleeding, including patients on anticoagulation.
- Similarly, routine use of aspirin for the sole purpose of primary prevention is best deferred in patients at low risk $(<5 \% 10$-year risk) and borderline risk ( $5 \%-7.5 \% 10$ year risk). In selected cases in patients at borderline risk such as those with a strong family history, clinicians could also consider risk enhancers such as coronary calcium in the discussion about the risks and benefits of starting aspirin.

- A shared decision to initiate aspirin in those at intermediate risk $(7.5 \%-20 \% 10$ year risk) could be considered for patients with risk enhancers such as an elevated coronary calcium score $(>100)$ or elevated lipoprotein(a) (>50 mg/dL).

- In patients ages 40 to 70 at high risk of atherosclerotic cardiovascular disease (> 20\% at 10 years), it is reasonable to consider starting low-dose aspirin as a shared decision if the patient is thought to be at low risk of bleeding.

- Finally, scarcity of data in those younger than 40 precludes any recommendation to guide the use of aspirin in this population. A shared decision on the benefit of aspirin in younger patients (age $<40$ ) is recommended in selected cases at high risk of atherosclerotic cardiovascular disease based on an elevated lifetime risk, strong family history, familial hypercholesterolemia, elevated coronary calcium score, or elevated lipoprotein(a).

- Patients with diabetes are a unique population at higher risk of cardiovascular events. Again, risk factor modification is the first step for primary prevention of cardiovascular disease in this population, including glucose control favoring medications with proven cardiovascular benefit. Patientclinician risk discussion is strongly recommended to decide on the added value of starting aspirin in patients with diabetes, weighing perceived benefits against risks to the patient at hand. It is best to defer aspirin use in those at high risk of bleeding, while starting aspirin is reasonable in those patients with diabetes with multiple additional risk factors or who have risk enhancers such as a coronary calcium score higher than 100 and elevated lipoprotein(a).

\section{Ultimately, a preventive regimen of aspirin must reduce the rate of cardiovascular events by an absolute magnitude that is clinically meaningful}




\section{CONCLUSION}

Despite the large amount of data, the role of aspirin in contemporary practice for primary prevention of cardiovascular disease remains debatable. In contrast to the modest benefit reported by older trials, the most recent trials largely challenged the benefit of aspirin in current practice. This is in great part explained, as anticipated, by improved best preventive practices (eg, blood pressure control, lipid management with statins, smoking cessation) that dilute the potential benefit from aspirin for primary prevention. ${ }^{46}$

Nonetheless, the existing evidence mainly comes from low-risk populations and fails to definitively ascertain the impact of aspirin in those at higher risk of cardiovascular disease. If aspirin use is to be considered for primary prevention, it must remain limited to selected patients at elevated risk of cardiovascular disease but low risk of bleeding. The use of risk enhancers such as elevated coronary calcium scores and elevated lipoprotein(a) may be useful to accurately identify such patients at the higher end of the risk spectrum.

Further studies are needed to determine the primary prevention subgroups that would benefit from low-dose aspirin.

\section{REFERENCES}

1. ISIS-2 Collaborative Group. Randomised trial of intravenous streptokinase, oral aspirin, both, or neither among 17187 cases of suspected acute myocardial infarction: ISIS-2. Lancet 1988; 332(8607):349-360. doi:10.1016/S0140-6736(88)92833-4

2. Sandercock PAG. The International Stroke Trial (IST): a randomised tria of aspirin, subcutaneous heparin, both, or neither among 19435 patients with acute ischaemic stroke. Lancet 1997; 349(9065):1569-1581. doi:10.1016/S0140-6736(97)04011-7

3. Lewis HD, Davis JW, Archibald DG, et al. Protective effects of aspirin against acute myocardial infarction and death in men with unstable angina: results of a Veterans Administration cooperative study. $\mathrm{N}$ Engl J Med 1983; 309(7):396-403. doi:10.1056/NEJM198308183090703

4. Gent M. A randomised, blinded, trial of clopidogrel versus aspirin in patients at risk of ischaemic events (CAPRIE). Lancet 1996; 348(9038):1329-1339. doi:10.1016/S0140-6736(96)09457-3

5. Collins R, Peto R, Hennekens $\mathbf{C}$, et al. Aspirin in the primary and secondary prevention of vascular disease: collaborative meta-analysis of individual participant data from randomised trials. Lancet 2009; 373(9678):1849-1860. doi:10.1016/S0140-6736(09)60503-1

6. Piepoli MF, Hoes AW, Agewall S, et al. 2016 European guidelines on cardiovascular disease prevention in clinical practice. Eur J Prev Cardiol 2016; 23(11):NP1-NP96. doi:10.1177/2047487316653709

7. Pearson TA, Blair SN, Daniels SR, et al. AHA guidelines for primary prevention of cardiovascular disease and stroke: 2002 update: consensus panel guide to comprehensive risk reduction for adult patients without coronary or other atherosclerotic vascular diseases. Circulation 2002; 106(3):388-391. doi:10.1161/01.CIR.0000020190.45892.75

8. Gaziano JM, Brotons $\mathbf{C}$, Coppolecchia $\mathbf{R}$, et al. Use of aspirin to reduce risk of initial vascular events in patients at moderate risk of cardiovascular disease (ARRIVE): a randomised, double-blind, placebo-controlled trial. Lancet 2018; 392(10152):1036-1046. doi:10.1016/S0140-6736(18)31924-X

9. ASCEND Study Collaborative Group, Bowman L, Mafham M, Wallendszus $\mathrm{K}$, et al. Effects of aspirin for primary prevention in persons with diabetes mellitus. N Engl J Med 2018; 379(16):1529-1539. doi:10.1056/NEJMoa1804988

10. McNeil JJ, Wolfe R, Woods RL, et al. Effect of aspirin on cardiovascular events and bleeding in the healthy elderly. N Engl J Med 2018; 379(16):1509-1518. doi:10.1056/NEJMoa1805819

11. McNeil JJ, Woods RL, Nelson MR, et al. Effect of aspirin on disabilityfree survival in the healthy elderly. N Engl J Med 2018; 379(16):14991508. doi:10.1056/NEJMoa1800722

12. McNeil JJ, Nelson MR, Woods RL, et al. Effect of aspirin on all-cause mortality in the healthy elderly. N Engl J Med 2018; 379(16):1519-1528. doi:10.1056/NEJMoa1803955

13. Arnett DK, Blumenthal RS, Albert MA, et al. 2019 ACC/AHA guideline on the primary prevention of cardiovascular disease. J Am Coll Cardiol
2019. doi:10.1016/j.jacc.2019.03.010

14. Peto R, Gray R, Collins R, et al. Randomised trial of prophylactic daily aspirin in British male doctors. Br Med J (Clin Res Ed) 1988; 296(6618):313-316. doi:10.1136/bmj.296.6618.313

15. Steering Committee of the Physicians' Health Study Research Group. Final report on the aspirin component of the ongoing Physicians' Health Study. N Engl J Med 1989; 321(3):129-135. doi:10.1056/NEJM198907203210301

16. Thrombosis Prevention Trial: randomised trial of low-intensity oral anticoagulation with warfarin and low-dose aspirin in the primary prevention of ischaemic heart disease in men at increased risk. Lancet 1998; 351(9098):233-241. pmid:9457092

17. Hansson L, Zanchetti A, Carruthers SG, et al. Effects of intensive bloodpressure lowering and low-dose aspirin in patients with hypertension: principal results of the Hypertension Optimal Treatment (HOT) randomised trial. HOT Study Group. Lancet 1998; 35(9118):1755-1762. doi:10.1016/s0140-6736(98)04311-6

18. Ridker PM, Cook NR, Lee IM, et al. A randomized trial of low-dose aspirin in the primary prevention of cardiovascular disease in women. N Engl J Med 2005; 352(13):1293-1304. doi:10.1056/NEJMoa050613

19. Sacco M, Pellegrini F, Roncaglioni MC, Avanzini F, Tognoni G, Nicolucci A. Primary prevention of cardiovascular events with low dose aspirin and vitamin E in type 2 diabetic patients: results of the Primary Prevention Project (PPP) trial. Diabetes Care 2003; 26(12):3264-3272. doi:10.2337/diacare.26.12.3264

20. Berger JS, Roncaglioni MC, Avanzini F, Pangrazzi I, Tognoni G, Brown DL. Aspirin for the primary prevention of cardiovascular events in women and men: a sex-specific meta-analysis of randomized controlled trials. JAMA 2006; 295(3):306-316. doi:10.1001/jama.295.3.306

21. Belch J, MacCuish A, Campbell I, et al. The prevention of progression of arterial disease and diabetes (POPADAD) trial: factorial randomised placebo controlled trial of aspirin and antioxidants in patients with diabetes and asymptomatic peripheral arterial disease. BMJ 2008; 337:a1840. doi:10.1136/bmj.a1840

22. Ikeda Y, Shimada K, Teramoto T, et al. Low-dose aspirin for primary prevention of cardiovascular events in Japanese patients 60 years or older with atherosclerotic risk factors: a randomized clinical trial. JAMA 2014; 312(23):2510-2520. doi:10.1001/jama.2014.15690

23. Ogawa H, Nakayama M, Morimoto T, et al. Low-dose aspirin for primary prevention of atherosclerotic events in patients with type 2 diabetes: a randomized controlled trial. JAMA 2008; 300(18):2134-2141. doi:10.1001/jama.2008.623

24. Fowkes FGR, Price JF, Stewart MCW, et al. Aspirin for prevention of cardiovascular events in a general population screened for a low ankle brachial index: a randomized controlled trial. JAMA 2010; 303(9):841848. doi:10.1001/jama.2010.221

25. Raju N, Sobieraj-Teague M, Hirsh J, O’Donnell M, Eikelboom J. Effect 


\section{SCHENONE AND LINCOFF}

of aspirin on mortality in the primary prevention of cardiovascular disease. Am J Med 2011; 124(7):621-629.

doi:10.1016/j.amjmed.2011.01.018

26. Raju N, Sobieraj-Teague M, Bosch J, Eikelboom JW. Updated metaanalysis of aspirin in primary prevention of cardiovascular disease. Am J Med 2016; 129(5):e35-e36. doi:10.1016/j.amjmed.2015.10.046

27. Mahmoud AN, Gad MM, Elgendy AY, Elgendy IY, Bavry AA. Efficacy and safety of aspirin for primary prevention of cardiovascular events: a meta-analysis and trial sequential analysis of randomized controlled trials. Eur Heart J 2019; 40(7):607-617. doi:10.1093/eurheartj/ehy813

28. Guirguis-Blake JM, Evans CV, Senger CA, O'Connor EA, Whitlock EP. Aspirin for the primary prevention of cardiovascular events: a systematic evidence review for the U.S. Preventive Services Task Force. Ann Intern Med 2016; 164(12):804-813. doi:10.7326/M15-2113

29. Whitlock EP, Williams SB, Burda BU, Feightner A, Beil T. Aspirin use in adults: cancer, all-cause mortality, and harms: a systematic evidence review for the U.S. Preventive Services Task Force. US Prev Serv Task Force Evid Synth 2015; (132):AHRQ Publication No. 13-05193-EF-1.

30. Whitlock EP, Burda BU, Williams SB, Guirguis-Blake JM, Evans CV Bleeding risks with aspirin use for primary prevention in adults: a systematic review for the U.S. Preventive Services Task Force. Ann Intern Med 2016; 164(12):826-835. doi:10.7326/M15-2112

31. Buse JB, Ginsberg HN, Bakris GL, et al. Primary prevention of cardiovascular diseases in people with diabetes mellitus: a scientific statement from the American Heart Association and the American Diabetes Association. Circulation 2007; 115(1):114-126. doi:10.1161/CIRCULATIONAHA.106.179294

32. American Diabetes Association. Cardiovascular disease and risk management: standards of medical care in diabetes 2018. Diabetes Care 2018; 41:S86-S104. doi:10.2337/dc18-S009

33. AAFP. Clinical preventive service recommendation. Aspirin use to prevent CVD and colorectal cancer. https://www.aafp.org/patient-care/ clinical-recommendations/all/aspirin-use-prevention.html. Accessed April 2, 2020.

34. USPSTF. Final update summary: aspirin use to prevent cardiovascular disease and colorectal cancer: preventive medication. http://www.uspreventiveservicestaskforce.org/Page/Document/UpdateSummaryFinal/ aspirin-to-prevent-cardiovascular-disease-and-cancer $\% 5 \mathrm{Cnhttp} / / / \mathrm{www}$. cancernetwork.com/colorectal-cancer/uspstf-supports-aspirin-colorectal-cancer-prevention. Published 2016. Accessed February 12, 2018

35. Mitchell JD, Paisley R, Moon P, Novak E, Villines TC. Coronary artery calcium and long-term risk of death, myocardial infarction, and stroke: the Walter Reed Cohort Study. JACC Cardiovasc Imaging 2018. doi:10.1016/j.jcmg.2017.09.003

36. Greenland P, Blaha MJ, Budoff MJ, Erbel R, Watson KE. Coronary calcium score and cardiovascular risk. J Am Coll Cardiol 2018; 72(4):434-
447. doi:10.1016/j.jacc.2018.05.027

37. Blankstein R, Chandrashekhar $\mathbf{Y}$. Extensive coronary artery calcifications: no longer primary prevention! JACC Cardiovasc Imaging 2020 13(1 pt 10):183-185. doi:10.1016/j.jcmg.2019.12.007

38. Blaha MJ, Cainzos-Achirica M, Greenland P, et al. Role of coronary artery calcium score of zero and other negative risk markers for cardiovascular disease: the Multi-Ethnic Study of Atherosclerosis (MESA). Circulation 2016. doi:10.1161/CIRCULATIONAHA.115.018524

39. Miedema MD, Duprez DA, Misialek JR, et al. Use of coronary artery calcium testing to guide aspirin utilization for primary prevention: estimates from the Multi-Ethnic Study of Atherosclerosis. Circ Cardiovasc Qual Outcomes 2014; 7(3):453-460. doi:10.1161/CIRCOUTCOMES.113.000690

40. Chasman DI, Shiffman D, Zee RYL, et al. Polymorphism in the apolipoprotein(a) gene, plasma lipoprotein(a), cardiovascular disease, and low-dose aspirin therapy. Atherosclerosis 2009; 203(2):371-376. doi:10.1016/j.atherosclerosis.2008.07.019

41. Rothwell PM, Cook NR, Gaziano JM, et al. Effects of aspirin on risks of vascular events and cancer according to bodyweight and dose: analysis of individual patient data from randomised trials. Lancet 2018; 392(10145):387-399. doi:10.1016/S0140-6736(18)31133-4

42. De Berardis G, Sacco M, Evangelista V, et al. Aspirin and Simvastatin Combination for Cardiovascular Events Prevention Trial in Diabetes (ACCEPT-D): design of a randomized study of the efficacy of low-dose aspirin in the prevention of cardiovascular events in subjects with diabetes mellitus treated with statins. Trials 2007; 8. doi:10.1186/1745-6215-8-21

43. Selak V, Jackson R, Poppe K, et al. Predicting bleeding risk to guide aspirin use for the primary prevention of cardiovascular disease. Ann Intern Med 2019. doi:10.7326/M18-2808

44. Hamon M, Lemesle G, Tricot $\mathbf{O}$, et al. Incidence, source, determinants, and prognostic impact of major bleeding in outpatients with stable coronary artery disease. J Am Coll Cardiol 2014; 64(14):1430-1436. doi:10.1016/j.jacc.2014.07.957

45. Yasuda S, Kaikita K, Akao M, et al; AFIRE Investigators. Antithrombotic therapy for atrial fibrillation with stable coronary disease. N Engl J Med 2019; 381(12):1103-1113. doi:10.1056/NEJMoa1904143

46. Meyer J, Arps K, Blumenthal RS, Martin SS. New data on aspirin use in the era of more widespread statin use. American College of Cardiology, September 28, 2018. https://www.acc.org/latest-in-cardiology/ articles/2018/09/28/08/08/new-data-on-aspirin-use-in-the-era-of-morewidespread-statin-use. Accessed March 25, 2020.

Address: A. Michael Lincoff, MD, Department of Cardiovascular Medicine J2-3, Cleveland Clinic, 9500 Euclid Avenue, Cleveland, OH 44195; lincofa@ccf.org 